\title{
Pilot Study to Determine Optimal Stent Duration Following Ureteroscopy: Three versus Seven days
}

\author{
Charles J. Paul Nathan A. Brooks George M. Ghareeb Chad R. Tracy \\ Department of Urology, The University of lowa, lowa City, IA, USA
}

\section{Key Words}

Ureteroscopy • Nephrolithiasis • Ureter • Stents •

Post-operative complications

\begin{abstract}
Background/Aims: Many providers elect to place a stent following ureteroscopy for nephrolithiasis, but little data exists on the optimal duration to leave a stent. We sought to determine whether there are any differences in post-operative outcomes for patients with a 3 versus 7-day stent following ureteroscopy. Methods: We retrospectively reviewed 247 patients who underwent unilateral ureteroscopy with lithotripsy, 79 of whom removed a stent with an extraction string at 3 or 7 days post-operatively. These 2 groups were compared with regard to demographic information, preoperative variables, and post-operative outcomes. Results: Of all patients, 33\% experienced a post-procedure related event (phone call, extra clinic visit, and emergency department visit) within 30 days of their procedure, 39\% of 3-day stent patients compared to $21 \%$ of 7 -day patients $(p=0.11)$. Within the 3 days following stent removal, 3-day stent patients were significantly more likely to have a post-procedure related event than 7 -day patients ( 23 vs. $3 \%, p=0.026$ ). Conclusion: One third of patients with a post-operative ureteral stent will seek medical care in the 30 days following ureteroscopy. Leaving a stent for 3 versus 7 days may lead to worse outcomes with regard to post-operative events and flank pain.

Copyright $\odot 2017$ S. Karger AG, Basel
\end{abstract}

\section{KARGER}

Fax +4161306 1234

E-Mail karger@karger.com Accessible online at:

www.karger.com www.karger.com/cur

\section{Introduction}

Despite American Urological Association recommendations to the contrary, indwelling ureteral stent placement is performed in up to $80 \%$ of patients following ureteroscopy [1-4]. Surveys of urologists reveal that $64 \%$ of urologists "always" leave a stent [5]. Stent placement is not without consequence and has been shown to lead to morbidity including flank pain, hematuria, dysuria, and lower urinary tract symptoms (LUTS) [6-10], as well as increased post-procedure related events (PREs) including phone calls, extra clinic appointments, and emergency department visits [11]. For patients who require cystoscopic stent removal, extraction is associated with additional risks, morbidity, cost, and time [12-14]. We have previously shown that the use of extraction strings decreases the pain of stent removal for patients without any significant difference in stent-related symptoms, morbidity, post-operative complications, or PREs $[6,11]$. Additionally, the ability to remove the stent at home via an extraction string improves convenience for the patient and allows for removal at specified times that are not dependent on provider schedule or patient-centered factors such as time away from work and time for travel [6].

Though stents are not indicated, most providers performing ureteroscopy continue to employ them. Since there are no current guidelines for the optimal timing of stent removal, practice patterns vary dramatically from several days to several weeks $[4,15,16]$. Since stent tol- 


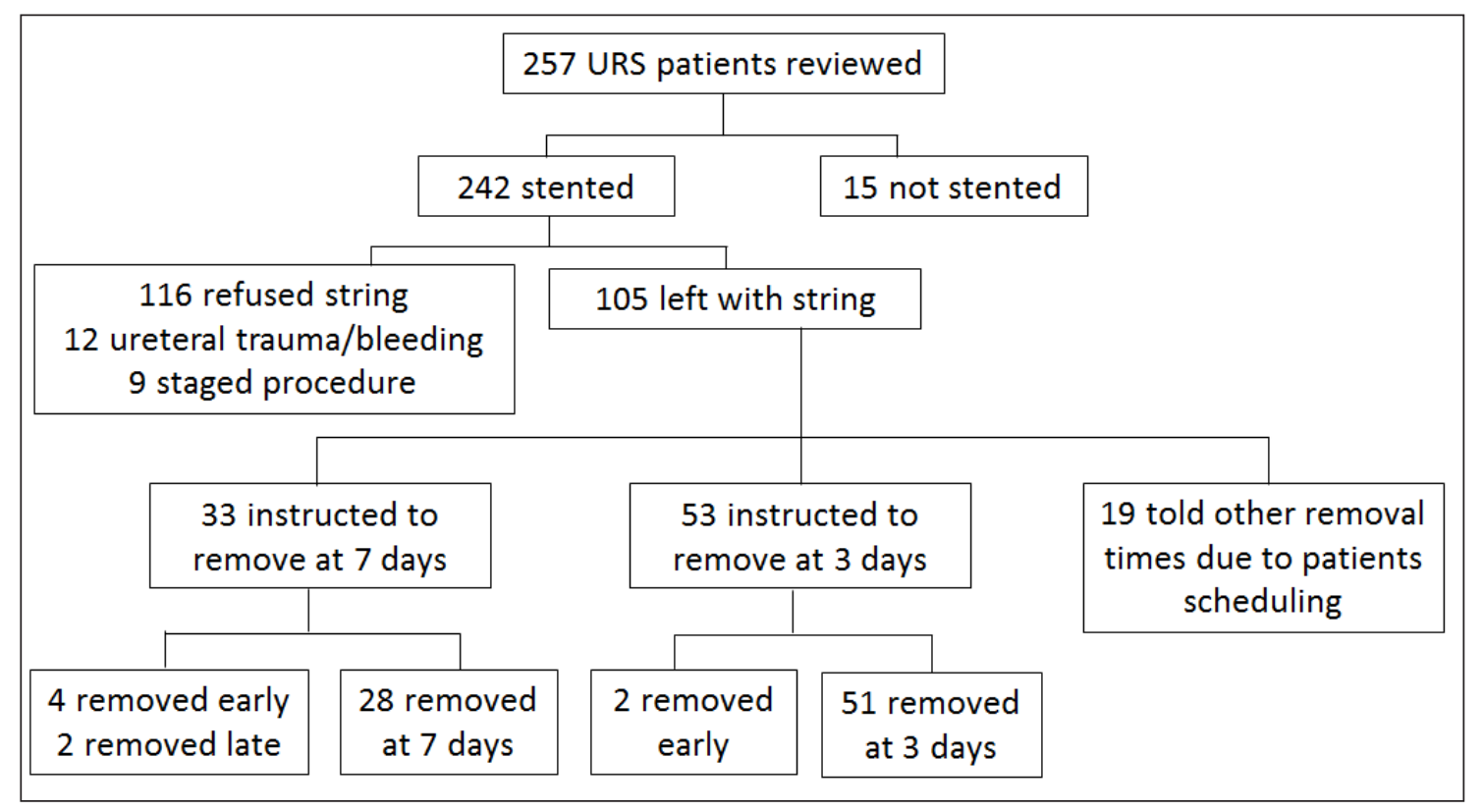

Fig. 1. Distribution of patient interventions. URS = Ureteroscopy.

erance is a known issue, it stands to reason that some of the morbidity and excess healthcare utilization could be mitigated with shorter stent duration. Despite the large number of studies on ureteroscopy and stents, no literature is currently available on optimal duration of stent placement. In order to determine the appropriate time for stent removal, we retrospectively reviewed consecutive patients undergoing ureteroscopy. We compared 2 cohorts of patients after implementing a practice change from 7-day stent dwell time to 3-day stent dwell time with regard to differences in PREs, symptomatic complaints, and post-operative complications.

\section{Materials and Methods}

Following Institutional Review Board approval, we retrospectively identified all patients who underwent ureteroscopy for nephrolithiasis by a single surgeon (C.R.T) from November 2010 through February 2016. Patients were excluded from analysis if they had a bilateral procedure, intra-operative ureteral injury, or if no extraction string was left in place. We limited our analysis to patients who were instructed to remove their stent on exactly post-operative day 3 or 7 and who did so within 1 day of their instructed date.

Ureteroscopy was performed with either a 7 French (Fr) semirigid ureteroscope for distal stones (Karl Storz, Tuttlingen, Germany) or a 7.95 or 9.9 Fr flexible ureteroscope (Olympus America, Center Valley, PA) for proximal or intrarenal stones. For renal calculi, access sheath utilization was based on the size of the patient's stone. All stents were 6 Fr with length ranging from 22 to $30 \mathrm{~cm}$ (Cook Medical; Bloomington, IN, USA). Prior to stent placement, the string was cut and re-tied with an air knot approximately $1 \mathrm{~cm}$ from the distal end of the stent as previously described $[6,17]$. Post-operatively, patients were instructed on the exact date to pull their stent at home. All patients were discharged on 2 weeks of tamsulosin, 3 days of antibiotics, as well as oxybutynin and narcotic pain medications prescribed as needed.

Prior to surgery, all patients undergoing unilateral ureteroscopy were offered the option of having an extraction string for self-retrieval versus returning for cystoscopic extraction. As there is a low, but significant, rate of inadvertent early dislodgement in patients with an extraction string, we opted to not leave a string in anyone with mucosal injury, complex anatomy such as pelvi-ureteric junction stenosis or horseshoe kidney, need for ureteral dilation, or suspected undiagnosed bacteriuria (based on intra-operative findings of cloudy or frankly purulent urine). For those with an extraction string, a practice change was implemented in May 2014 such that all patients were asked to remove the stent 3 days post-operatively. Follow-up included a clinic visit with kidneys, ureters, and bladder x-ray and renal ultrasound approximately 6 weeks after ureteroscopy.

Patients extracting their stents at 3 and 7 days were compared with regard to demographic information, pre-operative variables, and post-operative outcomes. Demographic variables included sex, age, American Society of Anesthesiologists Physical Status Classification (ASA) status, and date of service. Operative variables included stone burden, stone location, laterality, pre-operative stenting, access sheath use, planned stent duration, and actual stent duration. Stone burden was measured as the sum of the area of all stones seen on preoperative imaging. Our primary outcome 
Table 1. Patient characteristics

\begin{tabular}{|c|c|c|c|}
\hline Variable & $\begin{array}{l}\text { 3-Day } \\
\text { removal }\end{array}$ & $\begin{array}{l}\text { 7-Day } \\
\text { removal }\end{array}$ & $\mathrm{p}$ \\
\hline Number of patients, $\mathrm{n}$ & 51 & 28 & \\
\hline Mean age, year & 52 & 52 & 0.99 \\
\hline Sex, $n(\%)$ & & & 0.007 \\
\hline Male & $18(35 \%)$ & $19(68 \%)$ & \\
\hline Female & $33(65 \%)$ & $9(32 \%)$ & \\
\hline ASA status, n (\%) & & & 0.072 \\
\hline 1,2 & $42(82 \%)$ & $18(64 \%)$ & \\
\hline 3,4 & $9(18 \%)$ & $10(36 \%)$ & \\
\hline Prior stone episode, n (\%) & $37(73 \%)$ & $20(71 \%)$ & 0.91 \\
\hline Prior procedure on same side, n (\%) & $32(63 \%)$ & $15(54 \%)$ & 0.42 \\
\hline Stone burden, $\mathrm{mm}^{2}$ & & & 0.11 \\
\hline Median & 65.7 & 44.0 & \\
\hline Range & $4.0-313.8$ & $11.2-306.6$ & \\
\hline Stone location, n (\%) & & & 0.08 \\
\hline Renal & $33(65 \%)$ & $19(68 \%)$ & \\
\hline Ureter & $14(27 \%)$ & $3(11 \%)$ & \\
\hline Both & $4(8 \%)$ & $6(21 \%)$ & \\
\hline Laterality, n (\%) & & & 0.89 \\
\hline Right & $19(37 \%)$ & $10(36 \%)$ & \\
\hline Left & $32(63 \%)$ & $18(64 \%)$ & \\
\hline Pre-operative stenting, n (\%) & $14(27 \%)$ & $12(43 \%)$ & 0.17 \\
\hline $\begin{array}{l}\text { Mean pre-operative stenting dura- } \\
\text { tion, day }\end{array}$ & 21.2 & 28.7 & 0.31 \\
\hline Access sheath, n (\%) & $29(57 \%)$ & $22(79 \%)$ & 0.054 \\
\hline
\end{tabular}

measure was the presence of PREs, defined as either a phone call to the clinic, unscheduled clinic visit, or emergency department visit for a stent-related complaint within 30 days of the procedure. Additional outcome variables included gross hematuria, LUTS, dysuria, suprapubic pain, fever, additional serum or urine studies, early imaging, stent replacement, clinically insignificant residual fragments (CIRFs), and post-operative hydronephrosis. CIRFs were defined by follow-up imaging ( kidneys, ureters, and bladder x-ray/ultrasound) showing no stone larger than $2 \mathrm{~mm}$. Chi-squared test was used to determine statistical significance of categorical variables, and 2 sample $t$-test was used in analysis of continuous variables, as outcomes exhibited normal distribution. Statistical analysis was performed using SAS (version 9.4). Statistical significance was defined as having a $\mathrm{p}<0.05$.

\section{Results}

During the study period, 443 patients underwent ureteroscopy. Owing to the tertiary referral nature of our practice, only 257 (58\%) patients underwent unilateral ureteroscopy with lithotripsy from 2010 to 2016 and met the inclusion criteria for ureteral stent placement with a string. Stents were placed in 242 (94\%) and 105 (44\%) were left with an extraction string. The remaining 137 patients either refused an extraction string or had a more

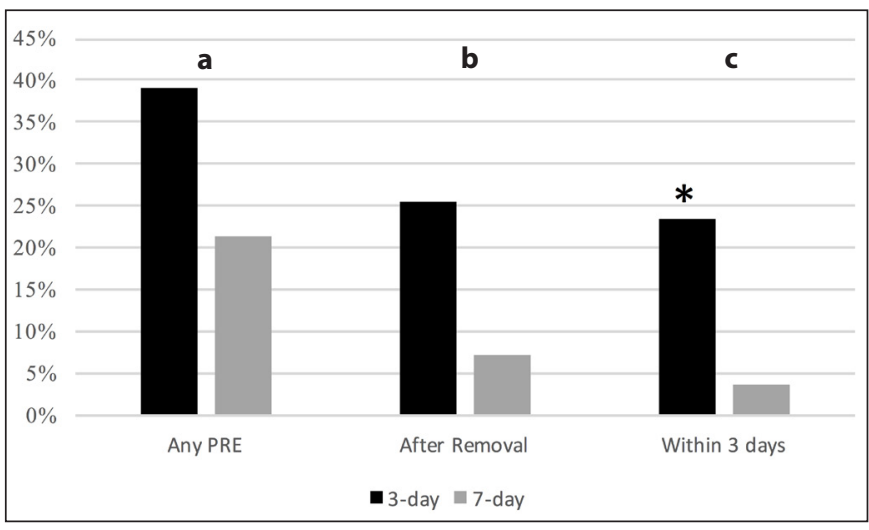

Fig. 2. a. Overall PRE occurrence in patients with 3-day and 7-day removal; b. PRE following stent removal; c. PRE within 3 days after stent removal.

complicated procedure than anticipated, precluding the use of an extraction string (fig. 1). Of patients with extraction strings, 53 were instructed to pull their stents at 3 days and 33 were instructed to pull their stents at 7 days after the procedure. The remaining 19 (all from the period prior to 3-day standardization) were instructed to remove stents at other intervals due to patient timing concerns. Ninety-six percent (51/53) of 3-day stent patients and $85 \%(28 / 33)$ of 7 -day patients removed their stents within 1 day of their instructed date and were included for further analysis. Six patients removed their stents early, 2 in the 3-day group and 4 in the 7-day, and were not included for further analysis. There was no difference between the 2 groups with respect to age, ASA status, prior stone episodes, prior procedures, stone burden, stone location, laterality, pre-operative stenting status, pre-operative stenting duration, or type of ureteroscope. There was a trend towards higher access sheath use in 7 -day patients $(\mathrm{p}=0.054)$, and women were significantly more likely to receive a 3-day stent than men $(\mathrm{p}=0.007$, table 1).

In total, 33\% of all patients experienced a PRE within 30 days of their procedure, $39 \%$ of 3-day stent patients compared to $21 \%$ of 7 -day patients ( $p=0.11$, fig. $2 a$ ). This was not affected by pre-procedure stent placement $(\mathrm{p}=0.19)$ or ureteral access sheath use $(\mathrm{p}=0.07)$. When considering PREs occurring after stent removal, $25 \%$ of 3-day stent patients had a PRE versus $7 \%$ of 7-day patients ( $p=0.062$, fig. $2 b$ ). Within the 3 days following stent removal, 3-day stent patients were significantly more likely to have a PRE than 7-day patients (23 vs. 


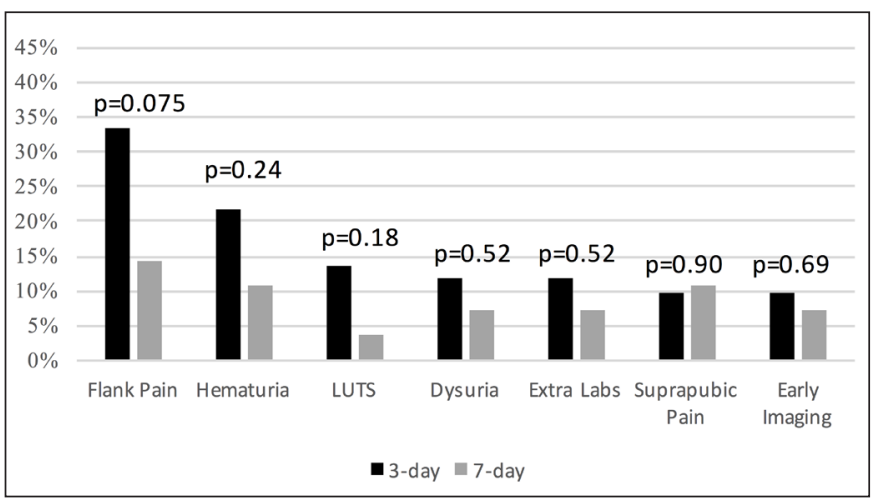

Fig. 3. Post-operative symptomatic complaints and diagnostics in 3-day and 7-day extraction groups.

$3 \%, \mathrm{p}=0.026$, fig. 2c). Sex did not affect PRE incidence $(\mathrm{p}=0.93)$. Patient phone calls accounted for the majority $(56 \%)$ of events.

The most reported symptoms were flank pain and gross hematuria (fig. 3). There was a trend toward increased flank pain in patients with a 3-day stent, with $33 \%$ of 3-day stent patients calling or presenting with flank pain versus $14 \%$ of 7 -day patients, $(p=0.075)$. No other symptoms approached significance, and no patients experienced proximal stent migration.

There was no difference between 3-day and 7-day patients in terms of CIRFs ( 88 vs. $86 \%, \mathrm{p}=0.82$ ). Two 3-day and one 7-day patient had urinary tract infections diagnosed post-operatively $(p=0.93)$. One patient in each group was admitted to the hospital for pain management following stent removal. No patients were found to have post-operative hydronephrosis during follow-up imaging.

\section{Discussion}

Ureteral stents are associated with significant morbidity. Approximately one third of patients experience a PRE following stent placement after uncomplicated ureteroscopy. If the indwelling stent is the cause for patients seeking care, shorter stent dwell times would lead to a decrease in PREs. Surprisingly, there was no significant difference in PREs between groups though more patients (39 vs. $21 \%$ ) experienced a PRE in the 3 -day group versus the 7-day group. We did identify a trend towards increased PREs after stent removal, with worse results in the 3 -day cohort ( 25 vs. $7 \%, \mathrm{p}=0.06)$. Furthermore, when looking just at the immediate 3 days following stent removal for both groups, those with a 3-day stent were significantly more likely to experience a PRE than those in the 7-day group $(p=0.02)$. One possible explanation for increasing post-stent removal PREs during this time may be due to relatively decreased ureteral dilatation or ongoing ureteral inflammation alleviated with the additional 4 days of stent dwell time.

Patients with stent discomfort may experience a wide variety of symptoms, and we sought to determine if stent dwell time mitigated certain symptoms. When comparing 3 versus 7 days, there was a trend toward increased flank pain in the 3-day cohort; however, this did not reach significance $(p=0.075)$. Although the number of patients reporting for an unscheduled emergency department or clinic visit was low, there was no difference in the need for extra laboratory or imaging studies between the 2 groups nor did any patient in either cohort require replacement of a stent following extraction.

While several studies have reported routine post-operative stenting is not required for uncomplicated ureteroscopy, the practice remains common and often related to surgeon preference $[1,18]$. Proponents of stenting reference uncertainty of who will and will not have complications, improvement in residual stone passage, and ease of stent removal versus emergent placement, particularly in patients who live far from the hospital $[4,7,19]$. Additionally, the majority of studies comparing stenting versus non-stenting do not involve cases utilizing ureteral access sheaths, limiting generalizability. Torricelli et al. [16] found that routine use of a stent following ureteroscopy with a ureteral access sheath led to lower pain scores and a reduced likelihood to seek medical assistance. It is our preference to perform ureteroscopy with an access sheath, which leads us to place a stent in the majority of patients, as was seen in this series. There was a trend toward more patients in the 7-day group having an access sheath compared to the 3-day group, such that we would have anticipated a higher incidence of post-operative pain in the 7-day group, rather than in the 3-day group. This again follows the trend that we have observed in increased PREs in the 3-day stent cohort.

There are limited studies evaluating the appropriate duration of stent placement following ureteroscopy. Chen et al. [20] randomized 60 patients undergoing ureteroscopy to stent placement for 3 days versus no stent. They found that patients with a 3-day stent were more likely to have significant discomfort than those without a stent. However, the study was limited to the fact that no patients underwent access sheath placement and all 
stones were $<10 \mathrm{~mm}$. In the study above by Torricelli et al. [16], evaluating stent symptoms following access sheath use, the stents were removed between 3 and 7 days post-operatively, but the effect of dwell time was not analyzed nor was there any comment on why patients were chosen for a particular stenting duration. Neither of these studies nor ours showed any effect of dwell time on CIRFs or rates of post-operative hydronephrosis.

The results of our study should be interpreted within the confines of its retrospective design. Although patients were not randomized, the decision to leave a stent for 3 days started on a specific date, which reduces several areas of potential bias. Similar to our prior work where we found $54 \%$ patients refused an extraction string [6], 48\% of patients in the current study were unwilling to remove their stent in this manner. There may be an underlying influence in patient preference for string that predicts post-operative recovery. However, as patients were not given a choice for 3 versus 7-day dwell time, we would expect that whatever differences there may be between patients electing for or refusing a string would not influence the outcomes of the current study. Clearly, many patients have a strong preference for having an extraction string (or not) and a rate of approximately $50 \%$ should be considered in any future prospective study on this topic.

Women were significantly more likely to receive a 3-day stent compared to men but were no more likely to experience a PRE. While we cannot definitively comment on the causes of these different rates between women and men, we do not feel that this biased our primary outcome measures as previous studies showed no difference in PRE rate in women compared to men [11]. Though PREs have previously been used as a method of quantitating post-operative outcomes, we acknowledge that PRE is not a validated instrument and relies on a patient's motivation to seek care. However, we have previously demonstrated, in our patient population, that PRE predicts outcomes nearly identical to that of the Ureteral Stent Symptom Questionnaire [6, 11, 21]. While patients treated at outside facilities might have been missed in PRE reporting, symptom occurrences and PRE rate were similar to other published series, and including phone calls helps to include patients having problems even if they do not seek care at our hospital. As this was not a prospective study, we were unable to evaluate symptoms on specific post-operative days. The change in stenting duration in this series followed a change in practice whereby following a specific date, stent duration for uncomplicated, routine ureteroscopy was decreased from 7 to 3 days. Because these patients were a similar group (no trauma, edema, or complications), this methodology should decrease selection bias that would come with deciding duration on a case by case basis. However, even when encountering favorable intra-operative findings such as minimal edema or little stone impaction, the results of this analysis gave us pause to prescribe a short stenting duration. Despite the limitations of the current study, based on these results, we no-longer instruct patients to remove stents at 3 days post-operatively, with a preference of dwell time of at least 5-7 days.

\section{Conclusion}

One in 3 patients with a post-operative ureteral stent will seek additional medical care in the 30 days following ureteroscopy. It does not appear that leaving a stent for 3 versus 7 days results in improved outcomes and may, in fact, lead to worse outcomes with regard to post-operative events and flank pain, particularly in the 3 days following stent removal. The pilot study provides a framework for future prospective analyses addressing stent dwell time and appropriateness of placement.

\section{Acknowledgements}

This study was funded by the Watts Family Fellowship in Urology within the Department of Urology at the University of Iowa. 


\section{References}

1 Auge BK, Sarvis JA, L'esperance JO, Preminger GM: Practice patterns of ureteral stenting after routine ureteroscopic stone surgery: a survey of practicing urologists. J Endourol 2007;21:1287-1291.

2 Merlo F, Cicerello E, Mangano M, Cova G, Maccatrozzo L: Stenting after ureteroscopy for ureteral lithiasis: results of a retrospective study. Arch Ital Urol Androl 2011;83:57-59.

3 Netto NR Jr, Ikonomidis J, Zillo C: Routine ureteral stenting after ureteroscopy for ureteral lithiasis: is it really necessary? J Urol 2001;166:1252-1254.

4 Damiano R, Autorino R, Esposito C, Cantiello F, Sacco R, de Sio M, D’Armiento M: Stent positioning after ureteroscopy for urinary calculi: the question is still open. Eur Urol 2004;46:381-387.

5 Dauw CA, Simeon L, Alruwaily AF, Sanguedolce F, Hollingsworth JM, Roberts WW, Faerber GJ, Wolf JS Jr, Ghani KR: Contemporary practice patterns of flexible ureteroscopy for treating renal stones: results of a Worldwide Survey. J Endourol 2015;29: 1221-1230.

6 Barnes KT, Bing MT, Tracy CR: Do ureteric stent extraction strings affect stent-related quality of life or complications after ureteroscopy for urolithiasis: a prospective randomised control trial. BJU Int 2014;113:605609.

7 Tang L, Gao X, Xu B, Hou J, Zhang Z, Xu C, Wang L, Sun Y: Placement of ureteral stent after uncomplicated ureteroscopy: do we really need it? Urology 2011;78:1248-1256.
8 Giannarini G, Keeley FX Jr, Valent F, Manassero F, Mogorovich A, Autorino R, Selli $\mathrm{C}$ : Predictors of morbidity in patients with indwelling ureteric stents: results of a prospective study using the validated Ureteric Stent Symptoms Questionnaire. BJU Int 2011;107: 648-654.

9 Joshi HB, Stainthorpe A, Keeley FX Jr, MacDonagh R, Timoney AG: Indwelling ureteral stents: evaluation of quality of life to aid outcome analysis. J Endourol 2001;15:151-154.

10 Tolley D: Ureteric stents, far from ideal. Lancet 2000;356:872-873.

11 Bockholt NA, Wild TT, Gupta A, Tracy CR: Ureteric stent placement with extraction string: no strings attached? BJU Int 2012;110 (11 pt C):E1069-1073.

12 Jeong YB, Doo AR, Park HS, Shin YS: Clinical significance of ureteral stent removal by flexible cystoscopy on pain and satisfaction in young males: a prospective randomised control trial. Urolithiasis 2016;44:367-370.

13 Kim DJ, Son JH, Jang SH, Lee JW, Cho DS, Lim CH: Rethinking of ureteral stent removal using an extraction string; what patients feel and what is patients' preference? : a randomized controlled study. BMC Urol 2015;15: 121.

14 Loh-Doyle JC, Low RK, Monga M, Nguyen MM: Patient experiences and preferences with ureteral stent removal. J Endourol 2015; 29:35-40.
15 Althaus AB, Li K, Pattison E, Eisner B, Pais V, Steinberg P: Rate of dislodgment of ureteral stents when using an extraction string after endoscopic urological surgery. J Urol 2015;193:2011-2014.

16 Torricelli FC, De S, Hinck B, Noble M, Monga M: Flexible ureteroscopy with a ureteral access sheath: when to stent? Urology 2014;83:278-281.

17 Barnes KT, Bing MT, Tracy CR: Video: Stent extraction strings after ureteroscopy, 2014, 2016.

18 Pengfei S, Yutao L, Jie Y, Wuran W, Yi D, Hao Z, Jia W: The results of ureteral stenting after ureteroscopic lithotripsy for ureteral calculi: a systematic review and meta-analysis. J Urol 2011;186:1904-1909.

19 Autorino R, Damiano R: Re: Ureteral stenting and urinary stone management: a systematic review G. Haleblian, K. Kijvikai, J. de la Rosette and G. Preminger J Urol 2008; 179: 424-430. J Urol 2008; 180:1573.

20 Chen YT, Chen J, Wong WY, Yang SS, Hsieh $\mathrm{CH}$, Wang CC: Is ureteral stenting necessary after uncomplicated ureteroscopic lithotripsy? A prospective, randomized controlled trial. J Urol 2002;167:1977-1980.

21 Joshi HB, Newns N, Stainthorpe A, MacDonagh RP, Keeley FX Jr, Timoney AG: Ureteral stent symptom questionnaire: development and validation of a multidimensional quality of life measure. J Urol 2003;169: 1060-1064. 